\title{
MicroRNA-335 and -543 suppress bone metastasis in prostate cancer via targeting endothelial nitric oxide synthase
}

\author{
QIZHONG FU, XIANFENG LIU, YING LIU, JIANXUN YANG, GUANGYAO LV and SHENGFANG DONG
}

Department of Urology, Zhongshan Hospital of Dalian University, Dalian, Liaoning 116001, P.R. China

Received January 18, 2015; Accepted August 13, 2015

DOI: $10.3892 / \mathrm{ijmm} .2015 .2355$

\begin{abstract}
Skeletal metastasis is the major problem in the management of prostate cancer (PCa). Even though the regulatory role of microRNAs (miRNAs) in the control of tumor metastases has been well described in numerous types of cancer, the importance in bone metastasis of $\mathrm{PCa}$ remains largely unknown. In the present study, the differentially expressed miRNAs were identified between the primary $\mathrm{PCa}$ and bone metastatic $\mathrm{PCa}$ samples by comparing their expression profiling using miRNA microarray, and 4 miRNAs ( $m i R-335,-543,-196$ and -19a) were noted to be significantly downregulated in bone metastasis compared with primary PCa. Among those, the downregulation of 2 miRNAs ( $m i R-335$ and -543) was confirmed in a total of 20 paired primary $\mathrm{PCa}$ and bone metastasis samples using reverse transcription-quantitative polymerase chain reaction. Using the online target prediction tool, endothelial nitric oxide synthase (eNOS) was found to be a shared target of $m i R-335$ and -543 , which was further verified using the luciferase assay. By examining the expression pattern of eNOS in primary PCa and skeletal metastatic samples, the mRNA and protein expression levels of eNOS were markedly upregulated in the metastatic samples. Furthermore, exogenous overexpression of $m i R-335$ and -543 significantly downregulated the expression level of eNOS, and substantially compromised the ability of migration and invasion in vitro. These findings suggested that $m i R-335$ and -543 are associated with bone metastasis of $\mathrm{PCa}$ and indicated that they may have important roles in the bone metastasis, which may also be clinically used as novel biomarkers in discriminating the different stages of human PCa and predicting bone metastasis.
\end{abstract}

\section{Introduction}

Prostate cancer ( $\mathrm{PCa}$ ) is the most common malignancy among men worldwide, and the natural course of the disease

Correspondence to: Dr Xianfeng Liu, Department of Urology, Zhongshan Hospital of Dalian University, 6 Jiefang Street, Dalian, Liaoning 116001, P.R. China

E-mail: liuxife111@163.com

Key words: miR-335, $m i R-543$, bone metastasis, prostate cancer is characterized by high heterogeneity, varying from indolent to highly aggressive cancer that spreads at an early stage of the disease and causes pain and ultimately mortality (1). Currently, the disease is stratified as low-, intermediate- and high-risk based on the prostate-specific antigen (PSA) level, Gleason score or clinical stage, whereas such stratification is deemed insufficient to predict the clinical outcome of $\mathrm{PCa}$, which is largely determined by the presence of metastasis, as even when grouped as high-risk, only $30 \%$ of the patients develop metastases and succumb due to their disease (2-4). The key problem in determining the clinical outcome of PCa is its characteristic to metastasize to bone, which essentially makes the disease incurable and leads to significant morbidity prior to patient fatality $(5,6)$. Therefore, it is of great clinical significance to determine the molecular mechanism underlying metastasis for the purpose of disease prevention, identification of new therapeutic targets and development of anti-metastatic therapies. Multiple steps, including cellular disengagement and degradation of the surrounding extracellular matrix, are believed to be involved in the formation of distant secondary bone tumors, and the total processes are under the control of multiple factors and molecular signaling pathways (7).

MicroRNAs (miRNAs or miRs) are short non-coding RNA strands with an average length of 22 nucleotides (8). Initially transcribed as RNA hairpins, miRNAs are processed into mature miRs that regulate the expression of genes at the post-transcriptional and the translational level by binding to complementary messenger RNA (9). To date, 1,000 human miRs have been identified and each of the miRNAs may target hundreds of genes (9), making it a complicated regulatory network of multiple molecular signaling pathways that are important for the control of human cell behavior (10). Increasing evidence indicates that miRNAs are significantly involved in the regulation of the development, progression and metastasis of a variety of types of cancer, as tumor suppressors or oncogenes (oncomiRs) (11). In PCa, several miRNAs have been reported as mediators of metastasis. The dysregulation of $m i R-221$ and $m i R-222$ is responsible for PCa progression, poor prognosis and the formation of metastasis (12). $m i R-21$ was also found to be overexpressed in $\mathrm{PCa}$, functioning as a major oncogenic regulator and contributing to tumor growth, invasiveness and metastasis $(13,14)$. Another study demonstrated that downregulation of $m i R-146 a$, as well as upregulation of its target, ROCK1, promote cell proliferation, invasion and metastasis in the PCa cells (15). 
To investigate the role of miRNAs in bone metastasis of $\mathrm{PCa}$, the miRNA expression profiles in primary and bone metastatic PCa were first examined and compared, and identified that $m i R-335$ and -543 is associated with bone metastasis. Furthermore, $m i R-335$ and -543 repressed migration and invasion in vitro, via targeting endothelial nitric oxide synthase (eNOS).

\section{Materials and methods}

Tissue samples. Tissue samples were collected from histologically confirmed $\mathrm{PCa}$ patients that were divided into two groups, primary PCa with $(n=20)$ and without $(n=15)$ bone metastasis. Primary PCa tissue samples were collected from all 35 enrolled patients (from prostatectomy or transurethral resection in the treatment of local prostate carcinoma) and skeletal metastatic tissue samples were collected from the 20 patients with $\mathrm{PCa}$ (from the surgical treatment of bone metastasis). The histological diagnosis was made by at least two experienced pathologists, and bone metastasis was diagnosed based on clinical symptoms and signs, as well as the imaging tests, including bone scan, computed tomography and magnetic resonance imaging. Those patients who had received neoadjuvant hormone, radiation or chemotherapy prior to the occurrence of tumor tissues were excluded from the study. The clinical characteristics and demographic data of the subjects, such as age, bone metastasis, total PSA level, free PSA level and the Gleason score, are described in Table I. The study was approved by the Institutional Ethical Board in the Zhongshan Hospital of Dalian University (Dalian, Liaoning, Chian), and written consent was obtained from each participant prior to the start of the study.

RNA extraction. All the tissue samples were embedded into paraffin blocks, cut into slices and placed in $1.5 \mathrm{ml}$ nuclease-free microcentrifuge tubes. Subsequently, the sliced tissue samples were deparaffinized three times in $1 \mathrm{ml} \mathrm{limo-}$ nene (Sigma-Aldrich, St. Louis, MO, USA), and washed with $1 \mathrm{ml} \mathrm{100 \%}$ ethanol twice prior to air drying at room temperature. Samples were subsequently incubated with digestion buffer (20 mM Tris-HCl, $10 \mathrm{mM}$ EDTA and 1\% SDS) and proteinase $\mathrm{K}$ (Merck, Whitehouse Station, NJ, USA) at $55^{\circ} \mathrm{C}$ overnight for the purpose of complete digestion. Subsequently, TRIzol reagent (Invitrogen, Carlsbad, CA, USA) was used to isolate the RNA following the manufacturer's instructions, and isolated RNA samples were resuspended in RNase-free water. Finally, RNA quality and quantity were evaluated using a NanoDrop (NanoDrop Technologies, Wilmington, DE, USA) and agarose electrophoresis.

Microarray analysis. The miRNA microarray chips were purchased from CapitalBio (CapitalBio Corp. Beijing, China), and each chip contained 924 probes in triplicate, corresponding to 677 human, 461 mouse and 292 rat miRNAs described in the miRNA registry (http://microrna.sanger.ac.uk; miRBase release 10.0,2007). The microarray analysis was carried out as described previously (16). The low-molecular-weight RNA was isolated using the polyethylene glycol solution precipitation method, and dephosphorylated by alkaline phosphatase (New England Biolabs, Ipswich, MA, USA) prior to fluorescently
Table I. Clinicopathological features of the prostate cancer patients with or without bone metastasis recruited in the study.

\begin{tabular}{lccc}
\hline Features & $\begin{array}{c}\text { PCa with bone } \\
\text { metastatsis } \\
(\mathrm{n}=20)\end{array}$ & $\begin{array}{c}\text { PCa without } \\
\text { bone metastatsis } \\
(\mathrm{n}=15)\end{array}$ & P-value \\
\hline Age, years & $65.2 \pm 7.23$ & $64.6 \pm 6.63$ & 0.638 \\
Pre-operative & $48.2 \pm 6.25$ & $50.5 \pm 7.51$ & 0.359 \\
PSA, ng/ml & & & \\
Gleason score & & & \\
$\leq 6$ & 2 & 3 & \\
7 & 4 & 4 & \\
8 & 6 & 4 & \\
9 & 5 & 3 & \\
10 & 3 & &
\end{tabular}

$\mathrm{PCa}$, prostate cancer; PSA, prostate-specific antigen.

labeling and precipitation. Subsequently, the RNA was resuspended in $20 \mathrm{ml}$ of hybridization buffer (36 SSC, $0.2 \%$ SDS and $15 \%$ formamide), and the hybridization was performed at $42^{\circ} \mathrm{C}$ overnight. The resultant chips were scanned with a double-channel laser scanner (LuxScan 10K/A), and the data was obtained from the TIFF images using LuxScan ${ }^{\text {TM }} 3.0$ software (both from CapitalBio Corp.). Raw data were normalized and analyzed using the significance analysis of microarrays (SAM, version 2.1; Stanford University, CA, USA) software.

Cell culture. PC-3, a metastatic PCa cell line, was purchased from American Type Culture Collection (Manassas, VA, USA) and cultured in F-12 medium supplemented with $10 \%$ fetal bovine serum (FBS) (both from Gibco, Eggenstein, Germany). Cells were grown at a humidified atmosphere of $5 \% \mathrm{CO}_{2}$ at $37^{\circ} \mathrm{C}$.

Reverse transcription-quantitative polymerase chain reaction $(R T-q P C R)$. RT-qPCR was used to confirm the expression level of mRNAs. Reverse transcription was performed using a superscript III first-strand synthesis system for an RT-PCR kit (Invitrogen), and RT-qPCR was performed on a Rotor-Gene 2000 Real-time Cycler detection system (Corbett Research, Sydney, Australia) supplied with analytical software, using an Express SYBR-GreenER qPCR Supermix Universal kit (Invitrogen), according to the manufacturer's instructions. U6 mRNA levels were used for normalization as an endogenous reference. The relative expression levels of $m i R-335, m i R-543$ and eNOS in the tissue samples were calculated using the $2^{-\Delta \Delta C T}$ method. Primer sets for the RT-qPCR are described as followed: $m i R-335$ forward, 5'-TCAAGAGCA ATAACGAAAAATGT-3' and reverse, 5'-GCTGTCAAC GATACGCTACGT-3'; miR-543 forward, 5'-GTGCTCGGT TTGTAGGCAGT-3' and reverse, 5'-GTGCCTTGTTTTGAT GGCAG-3'; eNOS forward, 5'-CCCTTCAGTGGCTGGTAC AT-3' and reverse 5'-CACGATGGTGACTTTGGCTA-3'; and U6 forward, 5'-CGCTTCGGCAGCACATATAC-3' and reverse, 5'-TTCACGAATTTGCGTGTCAT-3'. 
Western blotting. To evaluate the expression level of eNOS in the cell line with or without overexpressed $m i R-335$ and -543 , cells were seeded in 100-mm tissue culture dishes. After $48 \mathrm{~h}$, cells were washed with pre-chilled phosphate-buffered saline when the confluence reached $60-70 \%$, prior to being harvested in the lysis buffer [62.5 mmol/1 Tris- $\mathrm{HCl}$ (pH 6.8), $2 \%$ SDS, $10 \%$ glycerol, and 5\% 2- $\beta$-mercaptoethanol] (Sigma-Aldrich). Equal amounts of lysates were loaded for SDS-polyacrylamide electrophoresis, and the separated protein was transferred onto a PVDF membrane (Millipore, Billerica, MA, USA), followed by blocking in $5 \%$ non-fat milk for $1 \mathrm{~h}$ at room temperature, and incubating with the eNOS (Cat. no. sc-376751, dilution: 1:1,000) and $\beta$-actin (Cat.no. sc-47778, dilution: 1:10,000; both from Santa Cruz Biotechnology, Inc., Dallas, TX, USA) primary antibodies for $1.5 \mathrm{~h}$. Membranes were washed three times ( 5 min each) in Tris-buffered saline Tween 20 (TBS-T) buffer and incubated for $1 \mathrm{~h}$ at room temperature with horseradish peroxidase-conjugated secondary antibodies (Cat. no. sc-51625, dilution: 1:10,000; Santa Cruz Biotechnology, Inc.). Blots were washed three times (5 min each) in TBS-T and the signal was detected using an ECL system (Applygen, Beijing, China). $\beta$-actin was used to normalize the amount of each loading sample.

Scratch assay. The cell migration was determined by the scratch assay. The cells were cultivated to $90 \%$ confluence on 12-well plates and were transfected with the $m i R-335$ and $m i R-543$ mimics, and $m i R-335$ inhibitors or control. Subsequently, cell scrapers (Corning Inc., Corning, NY, USA) were utilized to scratch the confluent cells $24 \mathrm{~h}$ post-transfection. The procedures of cellular growth were subsequently observed. All the experiments were repeated in triplicate.

Transwell migration. The Transwell migration chambers were used to evaluate the PC-3 cell invasion. The cells were first seeded at a density of $1 \times 10^{5}$ cells in serum-free media on the upper chamber with the non-coated membrane $(8 \mu \mathrm{m}$ pore size; Millipore, Zug, Switzerland). The lower chamber contained F-12 medium with $20 \%$ FBS as a chemo-attractant. The cells in the upper chamber were discarded using cotton wool after $24 \mathrm{~h}$ and the migration cells in the lower chamber were counted using a microscope (Olympus, Tokyo, Japan). All the experiments were repeated in triplicate.

Luciferase assay. The full-length 3 ' untranslated region (3'UTR) of eNOS was amplified by PCR from genomic DNA and cloned into the pGL3-BS vector (Promega, Madison, WI, USA). The mutant construct of eNOS 3'UTR was generated using a QuickChange mutagenesis kit (Stratagene, Heidelberg, Germany). Co-transfection of the reporter vectors and miRs, mimics or controls was performed using Lipofectamine 2000 (Invitrogen). After 48 h, dual-luciferase activity was measured using the Dual-Luciferase ${ }^{\circledR}$ reporter assay system (Promega) according to the manufacturer's instructions.

Statistical analysis. Statistics were assessed using SPSS 20.0 (SPSS, Inc., Armonk, NY, USA). In the RT-qPCR and animal experiments, data were compared by Student's t-test. The association between downregulated miRNA expression and clinicopathological features in primary $\mathrm{PCa}$ with or without metastasis, and bone metastatic PCa was analyzed using the
Spearman's rank correlation test. In the metastasis assay-based experiments, the data were analyzed with one-way analysis of variance. For understanding the association between miRNAs, the significant correlations were determined using the Kendall's rank correlation test. $\mathrm{P}<0.05$ was considered to indicate a statistically significant difference.

\section{Results}

Identification of differentially expressed miRNAs between primary $P C a$ and bone metastasis using microarray analysis. To identify the differentially expressed miRNAs that could be responsible for the bone metastasis in $\mathrm{PCa}, 8$ pairs of primary and metastatic tissues (from the same patient) were collected and their expression profiles were compared using a microRNA microarray. A significantly upregulated expression of 14 miRNAs was revealed, as well as 4 downregulated miRNAs ( $m i R-543$, $-335,-196$ and $-19 a$ ) in bone metastasis compared with primary PCa (Fig. 1G). By cluster analysis, no miRNA expression pattern that was specific to the bone metastasis was identified. The online miRNA database was searched for the potential target genes that have been reported to be associated with metastasis in the literature. Notably, eNOS was a potentially shared target of $m i R-543$ and $m i R-335$, which were substantially downregulated in the present miRNA microarray analysis, by searching the online database www.targetscan.org (Fig. 1A and B). In addition, the 'seeds sequences' were replaced with the mutants in the 3'UTR of eNOS (Fig. 1C and D), and their interactions were tested with the microRNAs, finding that overexpression of $m i R-543$ and $m i R-335$ could significantly suppress the luciferase activity of those carrying wild-type 3'UTR of eNOS; however, no inhibitory effect was shown on the luciferase activity of those carrying the corresponding mutant (Fig. 1E and F). The data of the luciferase assay demonstrated that $m i R-543$ and $m i R-335$ could suppress the expression of eNOS by binding the specific 'seed sequence' in the 3'UTR of the gene.

Verification of the downregulation of $m i R-543$ and $m i R-335$ by RT-qPCR analysis in bone metastasis compared with primary $P C a$. To confirm the microarray data, RT-qPCR was conducted to evaluate the expression of the significantly downregulated miRNAs ( $m i R-543,-335,-196$ and -19a) in the independent samples of 20 primary $\mathrm{PCa}$ and their paired bone metastasis samples. Among them, 20 samples were paired samples of primary PCa and bone metastasis, including the 8 paired samples that have been involved in the miRNA microarray analysis. Subsequent to the quantification of the individual miRNA level in each sample and normalization to U6 expression, RT-qPCR data confirmed the downregulated expression of $m i R-543$, and $m i R-335$ in the bone metastatic tissues, respectively (Fig. 2A and B). The expression levels of $m i R-543$ and -335 were downregulated significantly in metastasis samples versus primary PCa. Although the expression of miR-196 and -19a was significantly downregulated in bone metastasis compared with in primary PCa samples in the microarray analysis, there were no statistically significant differences regarding the two miRs by RT-qPCR analysis (data not shown). Thus, the data indicated that there was a significant downregulation of miR-543 and -335 when PCa tumors metastasized to bone. As miR-543 and -335 levels were 
A WT eNOS 3'UTR (85-91)
Hsa-miR-335

C WT ENOS 3'UTR (85-91) Mut1 eNOS 3.UTR (85-91)
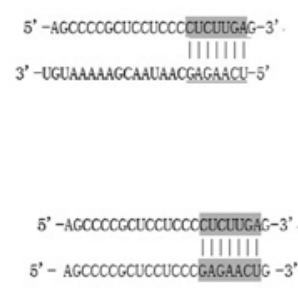

B

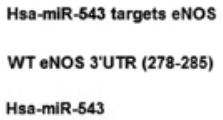

Hsa-miR-543

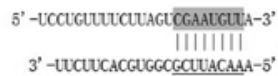

D WT ENOS 3'UTR (278-285) Mut2 eNOS 3'UTR (278-285)
E

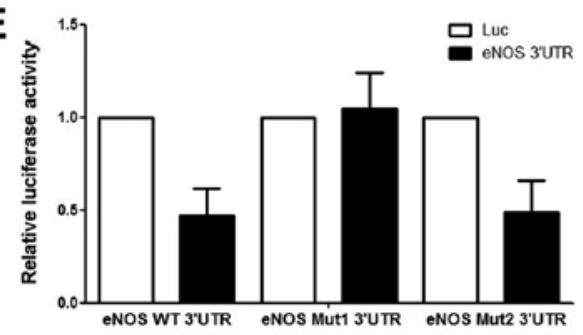

$\mathbf{F}$

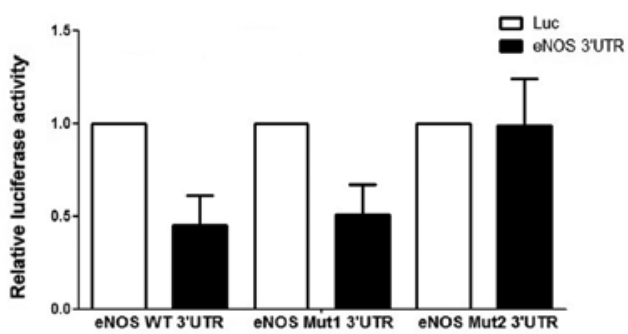

G

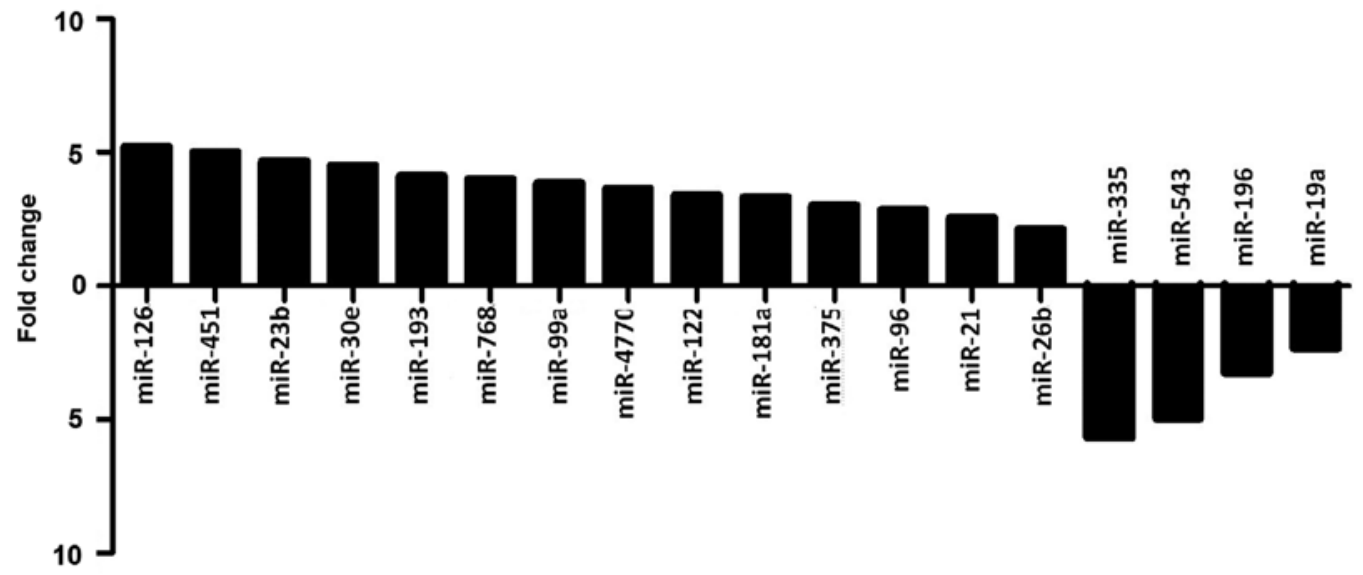

Figure 1. Schematic comparison between (A) $m i R$-335 and (B) $m i R-543$ and the 'seed sequence' in the 3'UTR of eNOS. Introduction of mutations to the (C) $m i R-335$ and (D) $m i R-543$ specific 'seed sequence' in the 3'UTR of eNOS. Luciferase activities in the (E) $m i R-335$ and (F) $m i R-543$ overexpressing cells transfected with wild-type or mutant 3'UTR of eNOS. (G) Fold change between the primary prostate cancer versus bone metastatic samples. 3'UTR, 3' untranslated region; eNOS, endothelial nitric oxide synthase; WT, wild-type; Mut, mutant.

downregulated in bone metastasis, we postulated that downregulation of $m i R-543$ and -335 may also be associated with an increased expression level of eNOS. Furthermore, the mRNA and protein expression patterns of eNOS were determined in the primary PCa and bone metastasis samples using RT-qPCR and western blot analysis. The mRNA level of eNOS was significantly upregulated in the bone metastasis compared with primary PCa (Fig. 2D), and consistently, the protein expression of eNOS was increased in the bone metastasis as well (Fig. 2C).

Firstly, a retrospective comparison was performed between the aforementioned 20 PCa patients with bone metastasis and 15 PCa patients without bone metastatis. The distribution of age in those patients with and without bone metastases was not significantly different. The expression of $m i R-335$ and -543 in 20 patients with bone metastases was significantly lower than that in the 15 patients without bone metastases $(\mathrm{P}=0.035$ and $\mathrm{P}=0.028$ ) (Fig. 3A and $\mathrm{B})$. Secondly, the mRNA and protein expression patterns of eNOS were determined in the primary PCa with and without bone metastasis samples using RT-qPCR and western blot analysis. The mRNA level of eNOS was significantly upregulated in primary $\mathrm{PCa}$ without bone metastasis compared with those with bone metastasis (Fig. 3D), and consistently, the protein expression of eNOS was increased in the PCa with bone metastasis as well (Fig. 3C). Thirdly, whether the presence of bone metastasis was associated with the total serum PSA levels or Gleason scores was evaluated, and no significant difference was identified (Table I). Finally, whether the expression levels of $m i R-335$ and -543 were correlated with total serum level and Gleason score in primary PCa was assessed, and there was no correlation between the expression of $m i R-335 /-543$ and total PSA level or Gleason score was noted (data not shown).

Upregulation of $\mathrm{miR}-335$ and -543 reduces the expression of eNOS and its effect on the skeletal aggressiveness of $P C-3$ cells. To investigate the role of $m i R-335$ and -543 in the control of PCa metastasis, scramble controls, $m i R-335$ and -543 mimics were transfected into PC-3 cells, which are characterized by bone metastasis. Fold changes in the relative expression of $m i R-335$ and -543 transfected PC-3 cell lines were much higher than that in the cells transfected with the control (Fig. 4A and B). Transfection of $m i R-335$ and -543 mimics could significantly suppress mRNA and protein 

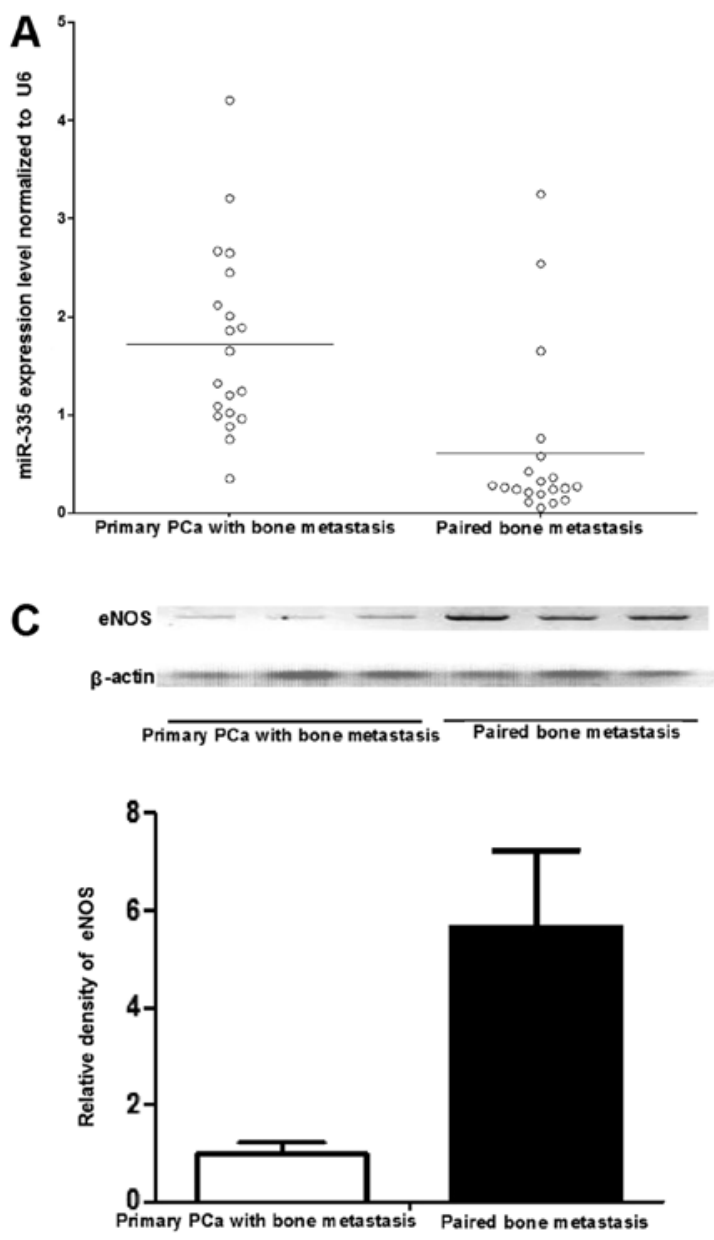

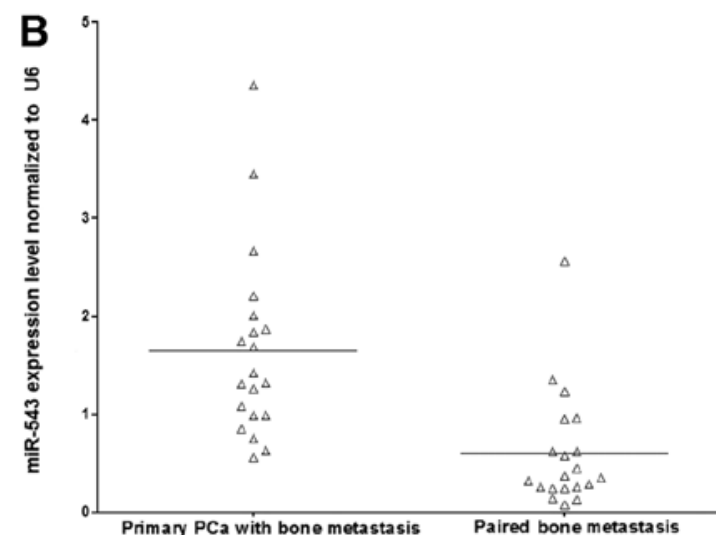

D

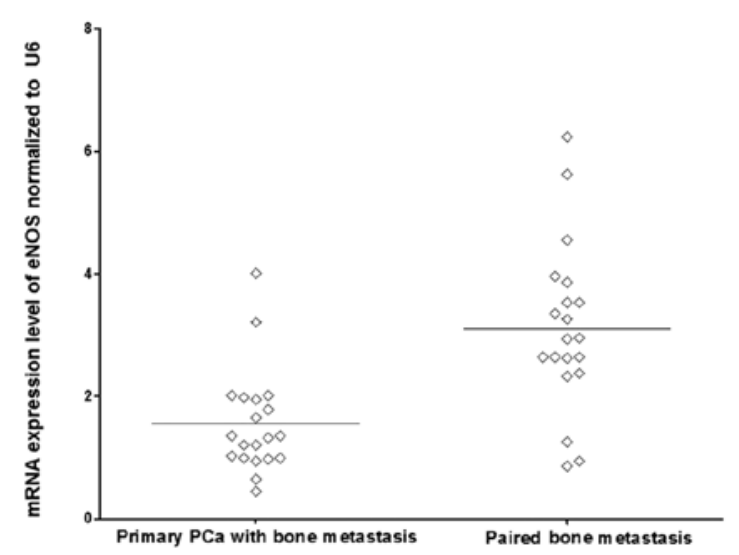

Figure 2. Comparison between primary PCa and the paired bone metastatic tissues regarding (A) $m i R-335$, (B) $m i R-543$, and $(\mathrm{C})$ protein (upper, selected western blot results; lower, densitometry analysis) and (D) mRNA expression level of eNOS. PCa, prostate cancer; eNOS, endothelial nitric oxide synthase.

expression levels of eNOS in PC-3 cells (Fig. 4C and D). Migration and invasion assays were performed in vitro. Notably, cell migration was observed by the wound healing assay and it was much slower when PC-3 cells were transfected with $m i R-335$ and -543 compared to the PC-3 cells transfected with the control (Fig. 5). The invasive property of PC-3 cells was examined by the Transwell-Matrigel penetration assay, which depicted that much fewer cells penetrated through the gel-membrane section when PC-3 cells were transfected with miR-335 and -543 compared to PC-3 cells transfected with the control (Fig. 6). The invasive property of PC-3 cells was significantly inhibited by $m i R-335$ and -543 , and was more evidently inhibited by $m i R-335$.

Downregulation of $\mathrm{miR}-335$ by introduction of its inhibitors promotes the expression of eNOS and its effect on the skeletal aggressiveness of $\mathrm{PC}-3$ cells. To further explore the role of $m i R-335$ and -543 in the control of PCa metastasis, scramble controls, $m i R-335$ and -543 inhibitors were transfected into PC-3 cells. Fold changes in the relative expression of $m i R-335$ transfected PC-3 cell lines were much lower than that of the cells transfected with the control, whereas the endogenous expression level of $m i R-543$ was too low to be further lowered (Fig. 7). Transfection of the miR-335 inhibitors could significantly promote mRNA and protein expression levels of
eNOS in PC-3 cells (Fig. 7). Migration and invasion assays were performed in vitro. Cell migration was observed by the wound healing assay and it was much slower when the PC-3 cells were transfected with $m i R-335$ and -543 compared to the PC-3 cells transfected with the control, in a time-dependent manner (Fig. 5).

\section{Discussion}

In the present study, the expression levels of $m i R-335$ and -543 were identified to be downregulated in skeletal metastatic PCa tumors compared with the primary tumors. Primary PCa patients without metastasis had significantly higher expression levels of $m i R-335$ and -543 compared to the patients with bone metastasis. The upregulation of $m i R-335$ and -543 reduced the aggressiveness of PC-3 cells, and suppressed the expression of eNOS. These results suggest that $m i R-335$ and -543 may have a significant role in the control of bone metastasis of PCa.

miRNAs have been repeatedly reported to be involved in the regulation of cancer metastasis and invasiveness. Among those, $m i R-335$ has been identified as a potent suppressor of tumor cell migration and invasion in multiple forms of cancer. For example, in breast cancer cells, upregulation of $m i R-335$ significantly decreased the invasive ability in a Boyden chamber assay and ectopic overexpression of the same 

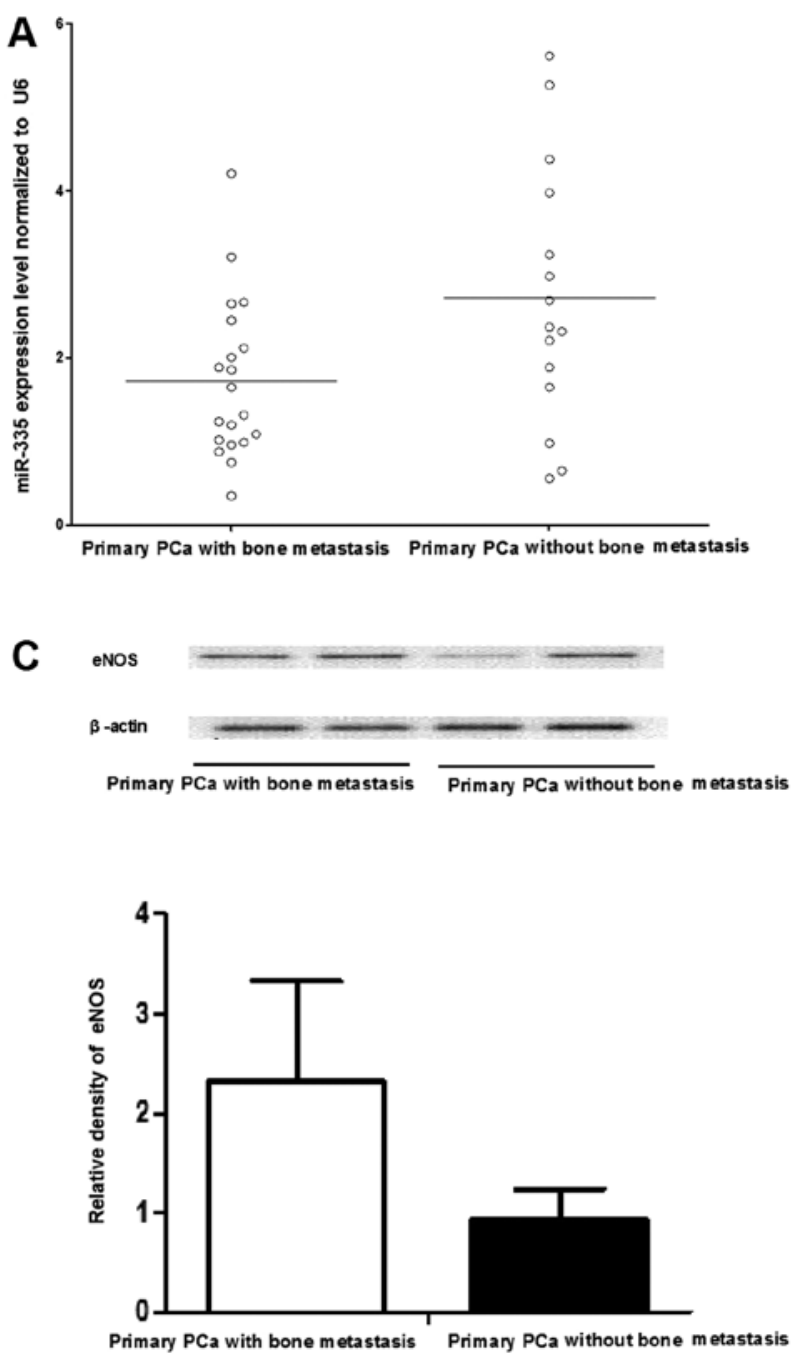

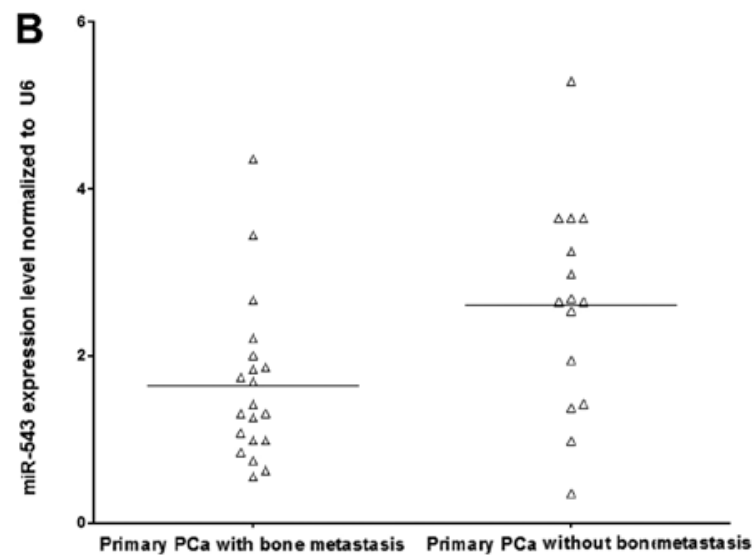

D

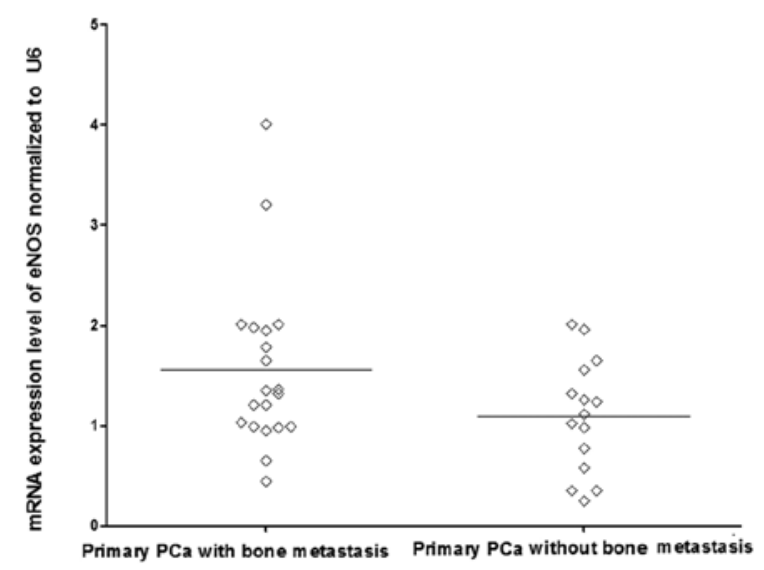

Figure 3. Comparison between primary PCa with and without bone metastatic tissues regarding (A) $m i R-335$, (B) $m i R-543$, and (C) protein (upper, selected western blot results; lower, densitometry analysis) and (D) mRNA expression level of eNOS. PCa, prostate cancer; eNOS, endothelial nitric oxide synthase.
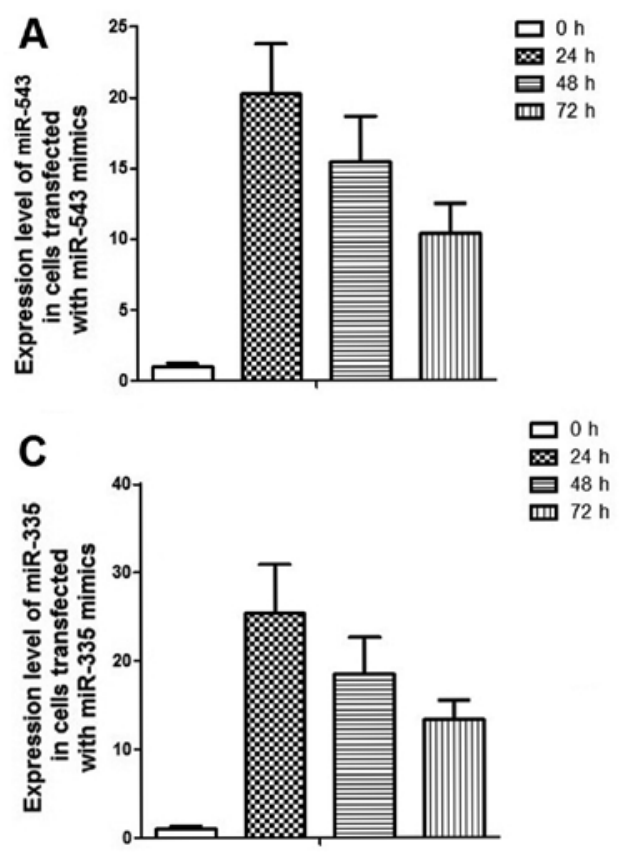

B

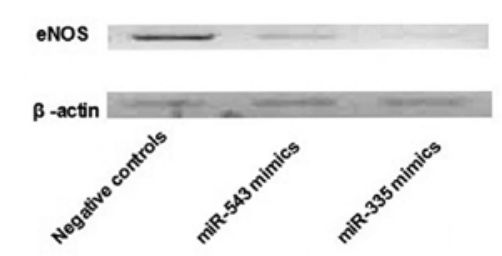

D

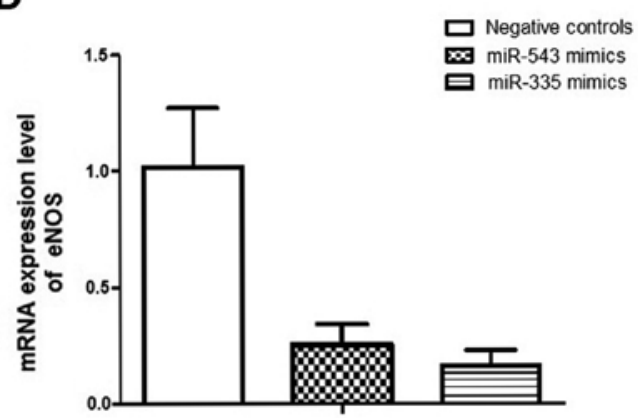

Figure 4. (A) Expression level of $m i R$-335 in PC-3 cells transfected with $m i R-335$ mimics. (B) Expression level of $m i R-543$ in PC-3 cells transfected with $m i R-543$ mimics. $m i R-335$ mimics and $m i R-543$ mimics decreased the (C) protein and (D) mRNA expression levels of eNOS. eNOS, endothelial nitric oxide synthase. 
A
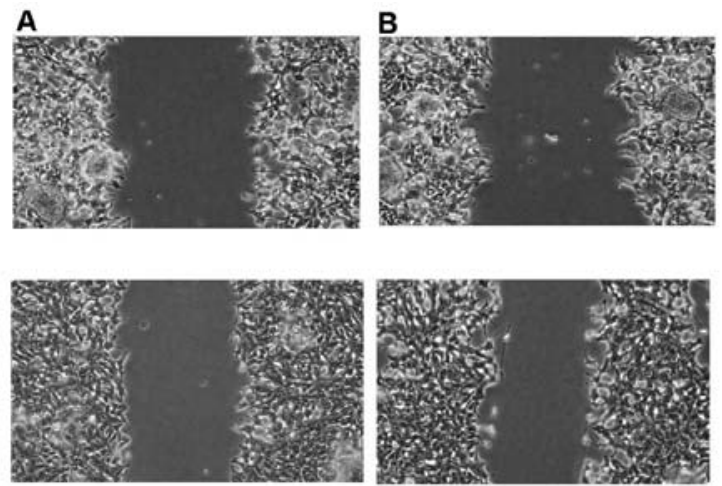
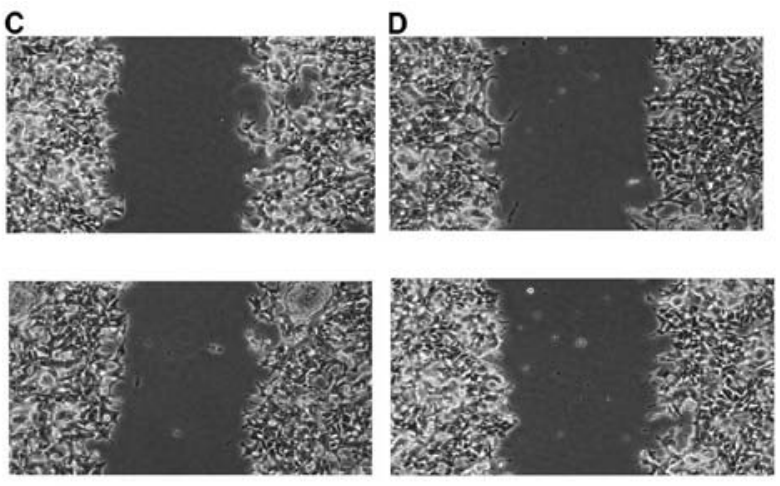

Figure 5. Migratory capability of PC-3 transfected with (A) the negative control (upper, 0 h; lower, 24 h), (B) miR-335 inhibitors (upper, 0 h; lower, 24 h), (C) miR-543 mimics (upper, $0 \mathrm{~h}$; lower, $24 \mathrm{~h}$ ) and (D) miR-335 mimics (upper, $0 \mathrm{~h}$; lower, $24 \mathrm{~h}$ ).

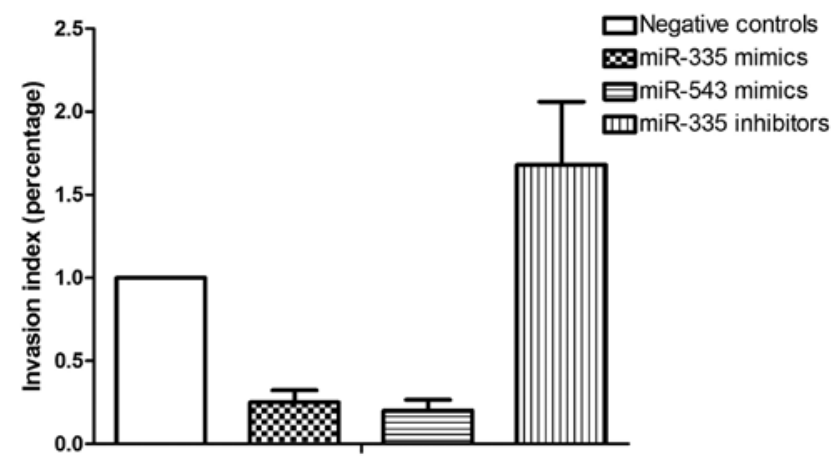

Figure 6. Invasive ability of PC-3 cells transfected with negative control, $m i R$-543 mimics, $m i R-335$ mimics and miR-335 inhibitors.

$\mathrm{miR}$ also reduced the formation of metastasis in lung (17). $\mathrm{Xu}$ et al (18) reported that $m i R$-335 may act as a potent metastasis suppressor in gastric cancer cells via directly targeting SP1 and indirectly modulating the Bcl-w-induced activation of the phosphoinositide 3-kinase-Akt-Sp1 signaling pathway, showing evidently altered expression of $m i R-335$ at different stages of gastric cancer and suggesting that the miR could be a therapeutic target and prognostic factor for gastric cancer. Using an RT-qPCR assay, miR-335 was reported to be downregulated in breast cancer tissues and sera (19). Similarly, the low expression of $m i R-335$ was significantly associated with adrenocortical carcinomas and acute myeloid leukemia $(20,21)$. Sorrentino et al (22) demonstrated that downregulation of $m i R-335$ may be responsible for the development of chemoresistance in ovarian cancer cells. $m i R$ - 335 has also been shown to indirectly regulate the myosin-driven motor activity of the cytoskeleton through modulating the expression levels of ROCK1, MAPK1 and LRG1 (23). Subsequently, the same research group extended this by showing that $m i R$-335 can directly regulate actin filament assembly and disassembly by targeting the Formin family of actin nucleators. Even though the majority of research data in the literature favors the tumor suppressive or anti-metastatic role of $m i R-335$, overexpression of miR-335 may also have an important role in the pathogenesis of numerous types of cancer, including colonic cancer, pediatric acute leukemia and multiple myeloma $(24,25,19)$, and such discrepancy could be attributed to the tissue specificity or ethnic diversity.
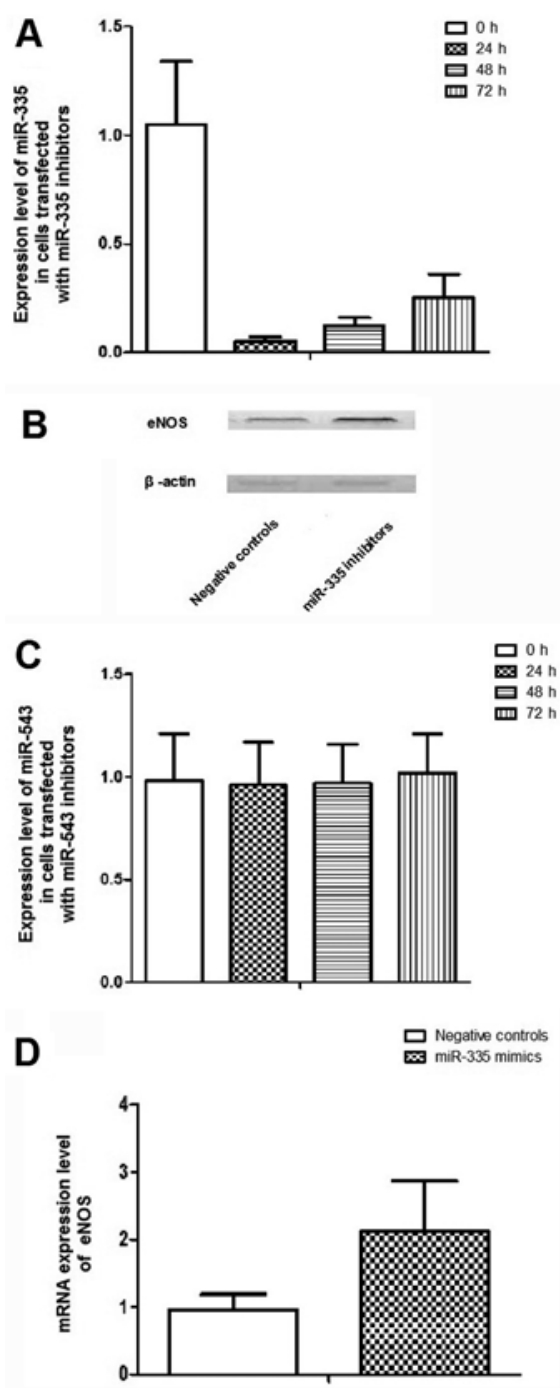

Figure 7. (A) Expression level of $m i R-335$ in PC-3 cells transfected with $m i R-335$ inhibitors. (B) Expression level of $m i R-543$ in PC-3 cells transfected with $m i R-543$ inhibitors. $m i R$-335 inhibitors increased the (C) protein and (D) mRNA expression levels of eNOS. eNOS, endothelial nitric oxide synthase.

To the best of our knowledge, there is only one study in the literature regarding the regulatory role of $m i R-543$ in malignant cells, where exogenous expression of $m i R-543$ in aggressive endometrial cancer cell lines was found to impair tumor cell 
migration and invasion, endogenous $m i R-543$ expression is substantially decreased in malignant versus normal endometrium tissue, and the levels of $m i R-543$ inversely correlated with mRNA levels of Focal adhesion kinase (FAK) and Twist homolog 1 (TWIST1), suggesting that FAK and TWIST1 may be effectors mediating the inhibitory effect of miR-543 on the migratory ability of tumor cells (26).

In the present study, 4 significantly downregulated miRNAs were identified in bone metastatic PCa samples using microarray analysis, and the downregulation of 2 among these was confirmed in an expanded pool of samples using RT-qPCR, showing that the expression of $m i R-335$ and -543 were lower in bone metastasis samples compared to primary PCa samples. Furthermore, the expression of $m i R-335$ and -543 were further upregulated in the sample tissues collected from the primary $\mathrm{PCa}$ without bone metastasis compared with the aforementioned primary PCa with bone metastasis, exhibiting the distinct expression patterns of $m i R-335$ and $m i R-543$ in the different types of tumor tissues: PCa without bone metastasis > PCa with bone metastasis > bone metastatic tissues.

Currently, the literature presents contradictory findings regarding the role of nitric oxide (NO) production in the control of cancer cell behavior. Certain studies have showed that elevated NO could facilitate vascularization in tumor cells by promoting angiogenesis, which is believed to be an important step in the formation of metastasis. It also could enhance mutagenesis through interacting with other free radicals to form cytotoxic compounds, such as peroxynitrite, leading to DNA damage (27). Simultaneously, the upregulation of NO that damages DNA may promote the growth of tumors via activating oncogenes and/or inhibiting tumor suppressor genes (28). By contrast, it was also reported that NOS activity was negatively correlated with tumor progression, which could be attributed to the ability of NO to protect cells from DNA damage by enhancing the activity of DNA-dependent protein-kinase catalytic subunit (DNA-PKcs), destroying tumor cells and stimulating apoptosis (29-31). Such discrepancy in the literature could be largely explained by the differential sensitivity of tumor cells to NO-mediated cytostasis or apoptosis, and clonal accumulation of NO-resistant or NO-dependent cells in different types of cancer (32). It was also reported that eNOS, mapped to $7 \mathrm{q} 35-7 \mathrm{q} 36$ in the human chromosome, may have an important role in the progression of PCa (33), and this is in line with the present results that exogenous overexpression of $m i R-335$ and -543 could synergistically downregulate eNOS in prostate cancer cells, and suppress the migratory capability, as well as the invasive ability, of the cancer cells by suppressing the gene.

In conclusion, the present findings suggest that $m i R-335$ and -543 may have significant roles in the bone metastasis of PCa and may be involved in the regulation of the migratory capability or invasive ability of the tumor cells. These two miRs may also be clinically used as novel biomarkers in discriminating different stages of human $\mathrm{PCa}$ and predicting the possibility of metastasis or even as therapeutic targets in bone metastasis of PCa.

\section{Acknowledgements}

The present study was fully sponsored by the Natural Science Foundation of China with grant no. 81373762.

\section{References}

1. Ferlay J, Autier P, Boniol M, Heanue M, Colombet M and Boyle P: Estimates of the cancer incidence and mortality in Europe in 2006. Ann Oncol 18: 581-592, 2007.

2. Petrovich Z, Lieskovsky G, Stein JP, Huberman M and Skinner DG: Comparison of surgery alone with surgery and adjuvant radiotherapy for $\mathrm{pT} 3 \mathrm{~N} 0$ prostate cancer. BJU Int 89: 604-611, 2002.

3. Spahn M, Joniau S, Gontero P, Fieuws S, Marchioro G, Tombal B, Kneitz B, Hsu CY, Van Der Eeckt K, Bader P, et al: Outcome predictors of radical prostatectomy in patients with prostate-specific antigen greater than $20 \mathrm{ng} / \mathrm{ml}$ : A European multi-institutional study of 712 patients. Eur Urol 58: 1-7, 10-11, 2010.

4. Wilt TJ, Brawer MK, Jones KM, Barry MJ, Aronson WJ, Fox S, Gingrich JR, Wei JT, Gilhooly P, Grob BM, et al; Prostate Cancer Intervention versus Observation Trial (PIVOT) Study Group: Radical prostatectomy versus observation for localized prostate cancer. N Engl J Med 367: 203-213, 2012.

5. Roodman GD: Mechanisms of bone metastasis. N Engl J Med 350: 1655-1664, 2004.

6. Mundy GR: Metastasis to bone: Causes, consequences and therapeutic opportunities. Nat Rev Cancer 2: 584-593, 2002.

7. Ye L, Kynaston HG and Jiang WG: Bone metastasis in prostate cancer: Molecular and cellular mechanisms (Review). Int J Mol Med 20: 103-111, 2007.

8. Catto JW, Miah S, Owen HC, Bryant H, Myers K, Dudziec E, Larré S, Milo M, Rehman I, Rosario DJ, et al: Distinct microRNA alterations characterize high- and low-grade bladder cancer. Cancer Res 69: 8472-8481, 2009.

9. Bartel DP: MicroRNAs: Target recognition and regulatory functions. Cell 136: 215-233, 2009.

10. Croce CM: Causes and consequences of microRNA dysregulation in cancer. Nat Rev Genet 10: 704-714, 2009.

11. Calin GA and Croce CM: MicroRNA signatures in human cancers. Nat Rev Cancer 6: 857-866, 2006.

12. Pang Y, Young CY and Yuan H: MicroRNAs and prostate cancer. Acta Biochim Biophys Sin (Shanghai) 42: 363-369, 2010.

13. Wang G, Wang Y, Feng W, Wang X, Yang JY, Zhao Y, Wang Y and Liu Y: Transcription factor and microRNA regulation in androgen-dependent and -independent prostate cancer cells. BMC Genomics 9 (Suppl 2): S22, 2008.

14. Si ML, Zhu S, Wu H, Lu Z, Wu F and Mo YY: miR-21-mediated tumor growth. Oncogene 26: 2799-2803, 2007.

15. Lin SL, Chiang A, Chang D and Ying SY: Loss of mir-146a function in hormone-refractory prostate cancer. RNA 14: 417-424, 2008.

16. Peng X, Guo W, Liu T, Wang X, Tu X, Xiong D, Chen S, Lai Y, Du H, Chen G, et al: Identification of miRs-143 and -145 that is associated with bone metastasis of prostate cancer and involved in the regulation of EMT. PLoS One 6: e20341, 2011.

17. Tavazoie SF, Alarcón C, Oskarsson T, Padua D, Wang Q, Bos PD, Gerald WL and Massagué J: Endogenous human microRNAs that suppress breast cancer metastasis. Nature 451: 147-152, 2008.

18. Xu Y, Zhao F, Wang Z, Song Y, Luo Y, Zhang X, Jiang L, Sun Z, Miao Z and Xu H: MicroRNA-335 acts as a metastasis suppressor in gastric cancer by targeting Bcl-w and specificity protein 1. Oncogene 31: 1398-1407, 2012.

19. Wang YX, Zhang XY, Zhang BF, Yang CQ, Chen XM and Gao HJ: Initial study of microRNA expression profiles of colonic cancer without lymph node metastasis. J Dig Dis 11: 50-54, 2010.

20. Marcucci G, Maharry K, Radmacher MD, Mrózek K, Vukosavljevic T, Paschka P, Whitman SP, Langer C, Baldus CD, Liu CG, et al: Prognostic significance of, and gene and microRNA expression signatures associated with, CEBPA mutations in cytogenetically normal acute myeloid leukemia with high-risk molecular features: A Cancer and Leukemia Group B Study. J Clin Oncol 26: 5078-5087, 2008.

21. Soon PS, Tacon LJ, Gill AJ, Bambach CP, Sywak MS, Campbell PR, Yeh MW, Wong SG, Clifton-Bligh RJ, Robinson BG, et al: miR-195 and miR-483-5p identified as predictors of poor prognosis in adrenocortical cancer. Clin Cancer Res 15: 7684-7692, 2009.

22. Sorrentino A, Liu CG, Addario A, Peschle C, Scambia G and Ferlini C: Role of microRNAs in drug-resistant ovarian cancer cells. Gynecol Oncol 111: 478-486, 2008.

23. Lynch J, Fay J, Meehan M, Bryan K, Watters KM, Murphy DM and Stallings RL: MiRNA-335 suppresses neuroblastoma cell invasiveness by direct targeting of multiple genes from the non-canonical TGF- $\beta$ signalling pathway. Carcinogenesis 33: 976-985, 2012. 
24. Ronchetti D, Lionetti M, Mosca L, Agnelli L, Andronache A Fabris S, Deliliers GL and Neri A: An integrative genomic approach reveals coordinated expression of intronic miR-335, miR-342, and miR-561 with deregulated host genes in multiple myeloma. BMC Med Genomics 1: 37, 2008.

25. Zhang $\mathrm{H}$, Luo XQ, Zhang P, Huang LB, Zheng YS, Wu J, Zhou $\mathrm{H}$, Qu LH, Xu L and Chen YQ: MicroRNA patterns associated with clinical prognostic parameters and CNS relapse prediction in pediatric acute leukemia. PLoS One 4: e7826, 2009.

26. Bing L, Hong $\mathrm{C}$, Li-Xin S and Wei G: MicroRNA-543 suppresses endometrial cancer oncogenicity via targeting FAK and TWIST1 expression. Arch Gynecol Obstet 290: 533-541, 2014.

27. Ying L and Hofseth LJ: An emerging role for endothelial nitric oxide synthase in chronic inflammation and cancer. Cancer Res 67: 1407-1410, 2007.

28. Lala PK and Chakraborty C: Role of nitric oxide in carcinogenesis and tumour progression. Lancet Oncol 2: 149-156, 2001.
29. Dhar A, Brindley JM, Stark C, Citro ML, Keefer LK and Colburn NH: Nitric oxide does not mediate but inhibits transformation and tumor phenotype. Mol Cancer Ther 2: 1285-1293, 2003.

30. Simeone AM, Colella S, Krahe R, Johnson MM, Mora E and Tari AM: N-(4-Hydroxyphenyl)retinamide and nitric oxide pro-drugs exhibit apoptotic and anti-invasive effects against bone metastatic breast cancer cells. Carcinogenesis 27: 568-577, 2006.

31. Fabbri F, Brigliadori G, Ulivi P, Tesei A, Vannini I, Rosetti M, Bravaccini S, Amadori D, Bolla M and Zoli W: Pro-apoptotic effect of a nitric oxide-donating NSAID, NCX 4040, on bladder carcinoma cells. Apoptosis 10: 1095-1103, 2005.

32. Jadeski LC, Chakraborty C and Lala PK: Role of nitric oxide in tumour progression with special reference to a murine breast cancer model. Can J Physiol Pharmacol 80: 125-135, 2002.

33. Qian J, Jenkins RB and Bostwick DG: Genetic and chromosomal alterations in prostatic intraepithelial neoplasia and carcinoma detected by fluorescence in situ hybridization. Eur Urol 35: 479-483, 1999. 